\title{
The Semantic Web as a Platform Against Risk and Uncertainty in Agriculture
}

\author{
Wilmer Henry Illescas Espinoza ${ }^{1}$, Alejandro Fernández ${ }^{2,3}$, and Diego Torres ${ }^{2,3,4}$ \\ ${ }^{1}$ Universidad Técnica de Machala, El Oro - Ecuador \\ willescas@utmachala.edu.ec \\ ${ }^{2}$ LIFIA, Fac.Informática, UNLP, 1900, La Plata, Argentina \\ \{Alejandro.fernandez, diego.torres\}@lifia.info.unlp.edu.ar \\ ${ }^{3}$ Comision de Investigaciones Científicas de Buenos Aires (CIC) \\ ${ }^{4}$ Departamento de Ciencia y Tecnología, UNQ, Bernal, Argentina.
}

\begin{abstract}
In this article, we discuss existing literature on DSS in agriculture, on DSS that use data available in the Semantic Web, and on Semantic Web initiatives focusing on agriculture information. Our goal is to assess the readiness of the Semantic Web as a platform to empower DSS that can keep risk and uncertainty in agriculture under control. Key agricultural activities targeted by DSS reported in literature are nutrient management, insect and pest management, land use and planning, environmental change and forecasting, and water and drought management. The most relevant use of Semantic Web in DSS, is in data analysis, as a means of making DSS more intelligent. There are initiatives to produce vocabularies and semantic repositories in the domain of agriculture. However, data and models are still isolated in specific domain repositories, and interoperability is still weak
\end{abstract}

Keywords: Decision Support Systems, Semantic Web, Agriculture

\section{Introduction}

We make decisions many times a day. The strategy we use depends on the importance of the decision. Intuitively, the more important the decision, the harder we need to think about it. Complexity, impact, uncertainty and risk are aspects that define the importance of the decision. The impact of a decision can be expressed as a function of its consequences (economic, health of people and environment, etc.). When factors not under control of the decision maker can negatively impact the result of the decision we talk about risk. The higher the probability and/or impact of a risk, the more thought needs to be put into making a decision.

When we do not count with all the information we need, or when the information we have is not completely accurate, we make our decisions under uncertainty [1]. Although we can decide on a given course of action, sometimes we can still be uncertain about its result. Frequently, the results of our actions depend on the response of what we act upon, or the actions taken by others. Uncertainty introduces risk, and consequently increases the complexity of our decisions. 
Mathematics and information technologies offer a rich variety of tool to help practitioners make decisions. These tools, generally called Decision Support Systems (DSS), are available in multiple domains. Our interest centers in agriculture. Decisions regarding planning, soil management, crop health, crop varieties, and commercialization were once made on the basis of common practices. However, globalization and constant climate change have become a source of complexity, uncertainty and risk. Moreover, decisions in any of the components of the agriculture value chain have far reaching consequences for the economy of the organizations, the health of people, and the environment.

DSS vary in complexity, strategies to support decision makers, and applicability. There are tools that support multiple stakeholders making decision that satisfy, as good as possible, the stakeholder's multiple constraints and requirements. There are tools that apply mathematical models to select from multiple, competing alternatives. There are tools that attempt to mimic how experts make decisions. There are tools that help decisions makers find, filter and analyze information.

Every decision we make requires information. The more relevant and high quality information we count on, the better are our chances to make a good decision. DSS can obtain information from sources internal or external to the organization. We are particularly interested in DSS that exploit information available on the World Wide Web. The Web is a constantly growing source of information, created by collaborating individuals around the world. However, most of the information published on the Web is targeted to humans, making the task of automated extraction and processing difficult and sometimes impossible. Luckily, there is a layer on the Web that has been specially prepared for machines. It is called the Semantic Web.

In this article, we review and integrate existing literature on DSS in agriculture, DSS that use data available in the Semantic Web, and Semantic Web initiatives focusing on agriculture information. Our goal is to assess the readiness of the Semantic Web as a platform to empower DSS that can keep risk and uncertainty in agriculture under control.

The remainder of this article is organized as follows. Section 2 introduces key concepts of Decision Support Systems and Semantic Web. DSS in Agriculture and DSS and the Semantic Web are introduced in Section 3 and Section 4 respectively. Section 5 discusses the presence of Agriculture Data in the Semantic Web. Finally, conclusions and challenges are described in Section 6.

\section{Background}

Decision Support Systems (DSS) have evolved since the 1970s. They are generally defined as computer-supported systems that use data and models to help system managers to analyze semi structured problems [2]. A DSS is "a computerbased system that aids the decision-making process" [3], and "an interactive, flexible, and adaptable computer based information system, specially developed for supporting the solution of a non-structured management problem for improved decision making" [2]. DSS are the subject of specialized conferences and workshops 
such as those organized by the European Working Group on Decision Support Systems [4], and the theme of specialized journals such as Decision Support Systems and Electronic Commerce [5].

In terms of application domains, DSS can be classified as: Personal (PDSS), Collaborative or Group (GSS), Negotiation Support Systems (NSS, Intelligent Decision Support Systems (IDSS), Knowledge Management Support Systems (KMSS), Business intelligence (BI), Data Warehousing (DW), or Enterprise Reporting and Analysis System (EIS). From the perspective of the types of core artifacts used to support decision making, they can be arranged as: Model-Driven DSS, Communication Driven DSS, Data Driven DSS, Document Driven DSS, and Knowledge Driven DSS.

The Semantic Web is a portion of the Web where the information is described in a machine-understandable format. The term Semantic Web was introduced by Berners-Lee as an evolution of the Web 2.0 [6] as a set of technologies that provides a better knowledge representation with the use of ontologies, software agents, and logic rules. Information in the Semantic Web is mainly described as RDF resources and OWL is the language to describe ontologies. RDF allows users to describe meaning and OWL increase them with inference rules and a specific domain. Additionally, SPARQL is a query language for RDF, which applications can access to RDF stores similar to SQL in a traditional database [7]. The Semantic Web is defined as a layered architecture. Each layer is built in a particular technology.

\section{DSS in Agriculture}

Mir and colleagues [8] provide a comprehensive overview of the role of DSS in agriculture. According to the authors, the key agricultural activities targeted by DSS reported in literature are nutrient management, insect and pest management, land use and planning, environmental change and forecasting, and water and drought management.

DSS focusing on nutrition aim at optimizing the use of fertilizers (which heavily impact cost) given various environmental and crop conditions. Some reported systems consider in addition the reuse farm by-products such as manure. Most commonly found DSS in this area are mathematical models of crop growth such as CERES [9], and expert systems such as Farm- $\mathrm{N}$ [10]. There is an ongoing trend towards making nutrition DSS available over the Web.

A similar review and classification was conducted by Manos and colleagues [11] for publications in the period 1987-2001. The majority of the articles $(59,2 \%)$ reported on advisory DSS that offered advice on strategic and tactical planning. In a smaller but still significant proportion, were articles reporting on control DSS (18.4\%), and forecasting DSS (14.3\%).

As early as 1993, [12] reported on the existence of 67 different expert systems dealing with plant protection. A key factor when dealing with insect and pest protection is to minimize the impact that chemicals have on the environment, while 
still being able to keep crops healthy. Disease expansion modelling and forecasting are commonly used decision support strategies in this area.

Land use and planning require dealing with complex trade-offs among the objectives of the various involved stakeholders (land-owners, farmers, governments, biodiversity protection organizations). Multi-objective models (especially goal programming) are frequently used mechanisms to implement DSS. In addition, planning support systems normally involve some form of geographic information systems functionality.

\section{DSS and the Semantic Web}

The most relevant use of Semantic Web in DSS context is the "use ontologies and rules as a means of making DSS more intelligent" in data analysis [13]. Much of the works could be viewed as continuation of the Expert Systems family of DSS, because they are using the SW standards such as RDF or OWL as an evolution of the use of raw data into knowledge representation $[14,15,16,17,18]$. The use of ontologies provides both a complete specialized domain knowledge model and a set of inference rules and querying to be applied in that model.

Semantic Web data can be used in different ways to power DSS; some approaches take advantages using RDF and OWL as formats to allow integration and systems communication $[19,20,21]$. Another improvement of the use of Semantic Web in DSS is the Ontology development, which is utilized on top of data to provide domain description and to integrate querying and reasoning $[19,20]$. The reuse of ontologies or vocabularies is promoted by the Linked Open Data movement.

The main challenges in the use of Semantic Web in DSS are related to make easier and flexible the integration methods of data and systems. Another challenge is how to capture knowledge naturally generated in informal contexts, and then make a matching with ontologies that describe it. The challenge here is to make this usable by a machine. In this direction, the study of the co-evolution between the social and the semantic web remarks some achievements in this direction [22].

Mendoza and colleagues [23] studied collaborative decision making using the Semantic Web as a platform. They identified challenges in three areas: the selection of vocabularies, the selection of data sources, and the retrieval and fusion of data. These challenges, if not tackled translate to an additional effort in the integration process, the need to discard data, and potential inconsistencies and lack of dataquality in the final product.

\section{Agricultural Data in the Semantic Web}

Sanders and colleagues propose a model to study the next generation of agricultural systems data, models and knowledge [24]. The model is a pyramid with data at the bottom, information on top of it, then knowledge and finally wisdom. According to this model, Linked Data conforms the base of the pyramid, the semantic web is at the information level, semantic technologies conform the 
knowledge level, and at the top we find Decision Support Systems. Applications constitute a "knowledge chain" that transform data into information, then into knowledge and finally into wisdom.

At the data layer we find raw data descriptions, for example, the controlled vocabulary AGROVOC by the Food and Agriculture Organization (FAO) [25]. Government and research have been the traditional sources of raw data (e.g., weather, statistics). The ubiquity of sensors and the new role of users as content producers introduce new actors in model definitions [24] and data collection. Examples of this change are the use of citizen science activities to collect data [26] or to validate data directly in the field $[27,28]$.

When data is aggregated with formal descriptors we look at the information layer. There, we find semantic repositories. The FAO-VEST/AgroPortal map [29] that includes $299 \mathrm{RDF}$ ontologies related to agriculture, crop and it lifecycle [30] ontologies belongs to the information layer. At this level we also find agriculture ontologies such as those described in [31,32]. When elements in the information layer are linked among them, the knowledge layer is generated [33].

The transformation from data to knowledge requires the definition of models, for example, nutrition or climatological models which combine knowledge representations, a software infrastructure, and hardware infrastructure. There are groups dedicated to model this processes such as the AGMIP project $[34,35]$ related to agricultural simulation and climate impacts, or the Geoshare ${ }^{1}$ group, which includes a broad range of agricultural data and analysis tools, among others.

DSS, Recommender Systems, Expert Systems and Advisory Systems are based on the relationships among different actors in the agriculture lifecycle, such as farmers and agricultural domain experts. These kinds of systems work at the knowledge and wisdom layers of the information chain. For their implementation, challenges still remain. Data and models are still isolated in silos, in specific domain repositories and the interoperability is still weak [24]; nevertheless, the use of ontologies could improve this by making possible the standardization of vocabulary, and data formats.

\section{Conclusions: opportunities and challenges}

The Semantic Web is a collaboratively constructed data repository. It depends on a sound theoretical model (ontologies and logic) and mature technology (the WWW). It shows characteristics of the collective intelligence [36] initiatives that made projects such as Wikipedia the center of today's information seeking.

As discussed in section 5, the number of vocabularies and ontologies available for the domain of agriculture is growing. However, initiatives that use Semantic Web data to support decision making in agriculture are rare.

${ }^{1}$ https://mygeohub.org/groups/geoshare last accessed on May 2017 
The Semantic Web, via initiatives like Linked Open Data, attempts to coordinate independent and previously disconnected efforts to standardize data representation formats, and vocabularies. Interoperability between applications depends on using compatible formats and unique identifiers to refer to the same entity. For example, to publish data that refers to the "Potato Beatle" (or Leptinotarsa decemlineata) its URI (http://aims.fao.org/aos/agrovoc/c_30034) should be used instead of any language specific string that would require human interpretation. Application developers need to learn these vocabularies and entity identifiers in order to create real Semantic Web aware systems.

The Semantic Web is an ecosystem that only flourishes when those that constitute it play according to its rules. Data generators (e.g., governmental agencies, research laboratories, technology providers) should participate in vocabulary standardization initiatives and publish data according to agreed vocabularies, aiming at the five stars of Linked Data ${ }^{2}$. Application developer need to learn and user existing vocabularies, consequently avoiding effort duplication. Whenever possible, application developers should contribute to making the Linked Data Cloud richer and up to date. Web site creators, even though their main primary audience consists of humans, should consider using approaches such as Microdata and $\mathrm{RDFa}$, to make the data in their web-sites available to software agents.

Decision making requires timely, relevant, high-quality information. For certain topics such as classification of crop varieties and insect species there are mature, trustable sources (e. g., Universities). However, data related to more dynamic phenomena such as climate, and to recent development such as innovative products and methods, require that multiple perspectives are represented and made available. Paradigmatic cases can often be found around topics where competing and often contradicting opinions are seen. In such scenarios, it is important to develop trustable participative decision making systems that account for evidence and offer provenance traceability.

Acknowledgments. Authors of this publication acknowledge the contribution of the Project 691249, RUC-APS: Enhancing and implementing Knowledge based ICT solutions within high Risk and Uncertain Conditions for Agriculture Production Systems (www.ruc-aps.eu), funded by the European Union under their funding scheme H2020-MSCA-RISE-2015.

\section{References}

1. Kochenderfer, M.J., Reynolds, H.J.D.: Decision making under uncertainty: theory and application. MIT press (2015).

2. Power, D.J.: Decision support systems: concepts and resources for managers. Greenwood Publishing Group (2002).

3. Turban, E.: Decision Support and Expert Systems: Management Support Systems. Prentice Hall PTR, Upper Saddle River, NJ, USA (1993).

${ }^{2}$ Linked Data - https://www.w3.org/DesignIssues/LinkedData.html . Last accessed on April 2017. 
4. Zaraté, P., Kersten, G. E., \& Hernández, J. E. (Eds.). (2014). Group Decision and Negotiation. A Process-Oriented View (Vol. 180). Cham: Springer International Publishing.

5. Decision Support Systems. Elsevier. ISSN: 0167-9236

6. Berners-Lee, T., Hendler, J., Lassila, O.: The Semantic Web. Sci. Am. 284, 34-43 (2001).

7. Hendler, J.: Web 3.0 Emerging. Computer (Long. Beach. Calif). 42, (2009).

8. Mir, S.A., Qasim, M., Arfat, Y., Mubarak, T., Bhat, Z.A., Bhat, J.A., Bangroo, S.A., Sofi, T.A.: Decision Support Systems in a Global Agricultural Perspective a Comprehensive Review. 7, 403-415 (2015).

9. Ritchie, J.T.: Model for predicting evaporation from a row crop with incomplete cover. Water Resour. Res. 8, 1204-1213 (1972).

10.Jorgensen, M.S., Detlefsen, N.K., Hutchings, N.J.: FarmN: A decision support tool for managing Nitrogen flow at the farm level. EFITA/WCCA. 25-28 (2005).

11.Manos, B., Ciani, A., Bournaris, T., Vassiliadou, I., \& Papathanasiou, J. (2004). A taxonomy survey of decision support systems in agriculture. Agricultural Economics Review, 5(2), 191-203.

12. Edwards-Jones, G.: Knowledge-based systems for crop protection: theory and practice. Crop Prot. 12, 565-578 (1993).

13. Blomqvist, E.: The use of Semantic Web technologies for decision support-a survey. Semant. Web. 5, 177-201 (2014).

14. Bouamrane, M.-M., Rector, A., Hurrell, M.: A hybrid architecture for a preoperative decision support system using a rule engine and a reasoner on a clinical ontology. In: International Conference on Web Reasoning and Rule Systems. pp. 242-253. Springer (2009).

15. Bouamrane, M.-M., Rector, A., Hurrell, M.: Experience of using owl ontologies for automated inference of routine pre-operative screening tests. In: International Semantic Web Conference. pp. 50-65. Springer (2010).

16. Buranarach, M., Supnithi, T., Chalortham, N., Khunthong, V., Varasai, P., Kawtrakul, A.: A semantic web framework to support knowledge management in chronic disease healthcare. In: Research Conference on Metadata and Semantic Research. pp. 164-170. Springer (2009).

17. Tao, C., Solbrig, H., Sharma, D., Wei, W.-Q., Savova, G., Chute, C.: Timeoriented question answering from clinical narratives using semantic-web techniques. Semant. Web-ISWC 2010. 241-256 (2010).

18. González, A.R., Fernandez, J., Jimenez, E., Mencke, M., Radzimski, M., Gomez, J.M., Alor-Hernandez, G., Posada-Gomez, R.: MEDFINDER-Using Semantic Web, Web 2.0 and Geolocation Methods to Develop a Decision Support System to Locate Doctors. In: WEBIST. pp. 288-293 (2009).

19. Baker, C.J.O., Shaban-Nejad, A., Su, X., Haarslev, V., Butler, G.: Semantic web infrastructure for fungal enzyme biotechnologists. Web Semant. Sci. Serv. Agents World Wide Web. 4, 168-180 (2006).

20. Bell, D., Nguyen, T.: Proximal business intelligence on the semantic web. In: Sustainable e-Business Management. pp. 145-159. Springer (2010). 
21. Bouamrane, M.-M., Rector, A., Hurrell, M.: Semi-automatic generation of a patient preoperative knowledge-base from a legacy clinical database. Move to Meaningful Internet Syst. OTM 2009. 1224-1237 (2009).

22. Torres, D.: Co-Evolution between Social and Semantic Web, (2014).

23. Mendoza, L., Zuccarelli, G., Díaz, A., Fernández, A.: The Semantic Web as a Platform for Collective Intelligence. In: CYTED-RITOS International Workshop on Groupware. pp. 255-262. Springer (2014).

24. Jones, J.W., Antle, J.M., Basso, B., Boote, K.J., Conant, R.T., Foster, I., Godfray, H.C.J., Herrero, M., Howitt, R.E., Janssen, S.: Toward a new generation of agricultural system data, models, and knowledge products: State of agricultural systems science. Agric. Syst. (2016).

25. Caracciolo, C., Stellato, A., Morshed, A., Johannsen, G., Rajbhandari, S., Jaques, Y., Keizer, J.: The AGROVOC linked dataset. Semant. Web. 4, 341-348 (2013).

26. Reges, H.W., Doesken, N., Turner, J., Newman, N., Bergantino, A., Schwalbe, Z.: COCORAHS: The evolution and accomplishments of a volunteer rain gauge network. Bull. Am. Meteorol. Soc. 97, 1831-1846 (2016).

27. Chaudhary, S., Bhise, M., Banerjee, A., Goyal, A., Moradiya, C.: Agro advisory system for cotton crop, (2015).

28. Walisadeera, A.I., Wikramanayake, G.N., Ginige, A.: Designing a Farmer Centred Ontology for Social Life Network. In: DATA. pp. 238-247 (2013).

29. Jonquet, C., Toulet, A., Arnaud, E., Aubin, S., Yeumo, E.D., Emonet, V., Pesce, V., Larmande, P.: AgroPortal: an open repository of ontologies and vocabularies for agriculture and nutrition data. In: GODAN Summit (2016).

30. Thunkijjanukij, A., Kawtrakul, A., Panichsakpatana, S., Veesommai, U.: Rice production knowledge management: criteria for ontology development. Thai J. Agric. Sci. 42, 115-124 (2009).

31. Xie, N., Wang, W., Yang, Y.: Ontology-based agricultural knowledge acquisition and application. In: International Conference on Computer and Computing Technologies in Agriculture. pp. 349-357. Springer (2007).

32. Pokharel, S., Sherif, M.A., Lehmann, J.: Ontology based data access and integration for improving the effectiveness of farming in nepal. In: Proceedings of the 2014 IEEE/WIC/ACM International Joint Conferences on Web Intelligence (WI) and Intelligent Agent Technologies (IAT)-Volume 02. pp. 319-326. IEEE Computer Society (2014).

33. Kang, J.C., Gao, J.L.: Application of Ontology Technology in Agricultural Information Retrieval. Adv. Mater. Res. 756-759, 1249-1253 (2013).

34. McDermid, S., Gowtham, R., Bhuvaneswari, K., Vellingiri, G., Arunachalam, L.: The impacts of climate change on Tamil Nadu rainfed maize production: a multi-model approach to identify sensitivities and uncertainties. Curr. Sci. 110, 1257 (2016).

35. Elliott, J., Kelly, D., Chryssanthacopoulos, J., Glotter, M., Jhunjhnuwala, K., Best, N., Wilde, M., Foster, I.: The parallel system for integrating impact models and sectors (pSIMS). Environ. Model. Softw. 62, 509-516 (2014).

36. Malone, T.W., Bernstein, M.S.: Handbook of collective intelligence. MIT Press (2015) 